\title{
CDC5L Promotes hTERT Expression and Colorectal Tumor Growth
}

\author{
Jia Lia Ningning Zhang ${ }^{a}$ Rui Zhang ${ }^{c}$ Longmei Sun ${ }^{a}$ Wendan Yu ${ }^{a}$ Wei Guo \\ Yingying Gao Mei Lia Wei Liu ${ }^{a}$ Pin Lianga Wuguo Deng ${ }^{b}$ Xiaonan Cui ${ }^{a}$ \\ aThe First Affiliated Hospital and Institute of Cancer Stem Cell, Dalian Medical University, Dalian, \\ bSun Yat-sen University Cancer Center; State Key Laboratory of Oncology in South China, Collaborative \\ Innovation Center for Cancer Medicine, Guangzhou, 'Department of colorectal surgery, Cancer Hospital \\ of China Medical University, Liaoning Cancer Hospital \& Institute, Liaoning, China
}

\author{
Key Words \\ $\mathrm{CDC} 5 \mathrm{~L} \cdot \mathrm{hTERT} \cdot \mathrm{CRC} \cdot$ Migration $\bullet$ Prognosis
}

\begin{abstract}
Background/Aims: Colorectal cancer (CRC) is the third leading cause of cancer-related death worldwide because the survival rate remains low. Cell division cycle 5 -like (CDC5L) is highly expressed in some cancer cells, but the mechanism requires clarification. Human telomerase reverse transcriptase (hTERT) plays important roles in CRC. Methods: This study aimed to identify a link between CDC5L and hTERT and to determine their effects on the signaling pathways, migration and prognosis of CRC cells. We first treated LoVo cells with biotin-labeled hTERT and identified CDC5L. Then, pulldown and ChIP assays were used to verify whether CDC5L was a promoter of hTERT. The roles of CDC5L and hTERT in cell growth and migration were studied using siRNA in vivo and in vitro. 130 human CRC specimens were analyzed using immunohistochemistry. Western blot and wound scratch analyses were used to determine the signaling pathway for CDC5L-mediated activation of CRC growth and migration. Results: We identified CDC5L as a new hTERT promoter-binding protein. Clinically, CDC5L and hTERT expression levels were key factors in the prognosis of CRC patients. CDC5L knockdown inhibited tumor growth by down-regulating hTERT expression, and CDC5L was shown to be a transcriptional activator of hTERT in a luciferase reporter assay. Conclusion: Altogether, the above results demonstrated that CDC5L was positively correlated with hTERT as a key promoter of CRC cells. To some extent, our findings suggest that CDC5L may serve as a novel therapeutic target for human colorectal cancer.

\section{Introduction}

Colorectal cancer (CRC) is one of the most common cancers in men and women. Approximately 376,300 newly diagnosed CRC cases and 191,000 associated deaths occurred 
in China in 2015 [1]. The incidence of CRC in China was 29.4 cases per 100,000 people in 2009 , which accounted for $10 \%$ of the newly diagnosed cancer cases in this country [2,3]. A rapid increase in the incidence of CRC in previously low risk countries, including countries in Asia and Africa, has been reported; this increased incidence is most likely linked to changes in diet and habits that include a move toward the so-called Western lifestyle [4]. Over the last decade, the availability of combination chemotherapy and targeted agents has improved the median survival time of patients with metastatic colorectal cancer (mCRC) $[5,6]$. Targeting the mitotic stage of the cell cycle is increasingly recognized as an effective approach for cancer therapy $[7,8]$. Research has focused primarily on alternative targets for antimitotic drugs that have low dose-limiting toxicities, which are foundational to the paradigm shift toward personalized cancer therapy [9].

Cell division cycle 5-like (CDC5L), which is related to Schizosaccharomyces pombe cdc5p and Saccharomyces cerevisiae CEF1, is essential for G2 progression and mitotic entry [10-13]. Overexpression of CDC5L in mammalian cells causes a shorter G2 phase and reduced cell size [10]. In contrast, knockdown of CDC5L in tumor cells results in dramatic mitotic arrest and chromosome misalignments, which eventually cause mitotic catastrophe [9]. CDC5L proteins in yeast and mammals are core components of the spliceosome complex and are essential for pre-mRNA splicing [14-21]. CDC5L plays an important role in the DNA damage response following exposure to genotoxic agents [22]. Furthermore, CDC5L is involved in kinetochore-microtubule attachment and DNA damage repair by regulating the expression and splicing efficiency of certain genes [9]. CDC5L is also highly expressed in some human somatic tumors, such as gliomas, osteosarcoma and hepatocellular carcinoma, and CDC5L genomic DNA amplification has been identified frequently in osteosarcoma [23]. These studies indicate that CDC5L may be a cancer-promoting protein. However, additional studies are needed to evaluate the role of this protein and its mechanisms in the development of CRC.

Human telomerase reverse transcriptase (hTERT) is an important component of human telomerase, which synthesizes telomeric DNA, lengthens chromosomes and maintains chromosomal stability, ultimately leading to cellular immortality [24]. hTERT is not expressed in most human somatic cells but is commonly overexpressed in a wide range of human cancers [25,26], including colorectal cancer [27]. The regulation of hTERT expression at the transcriptional level is a major mechanism in cancer development [28]. In reports comparing hTERT expression in normal and cancer cells, hTERT gene promoter activity was increased in cancer cells with activated hTERT expression. This observation is largely attributed to several key transcription factors in tumor cells that can up-regulate hTERT transcription [29]. To identify these potentially critical unknown factors, we successfully established a screening system that combined a streptavidin-agarose pulldown assay and high throughput proteomics [30]. One of the proteins identified using this systematic approach was CDC5L, which was expressed in hTERT promoter probe-protein complexes in the nuclear protein fractions prepared from colon cancer cells. In this study, we showed that low CDC5L expression inactivated the hTERT promoter, which subsequently inactivated hTERT transcription. We examined the effects of CDC5L on colon cancer cell proliferation and migration and identified the underlying molecular mechanisms in vitro and in vivo. We also studied the clinical association between CDC5L and hTERT in colorectal cancer tissue samples. Our results support our initial discovery that CDC5L may act as an important regulator of hTERT expression and participate in the development of CRC. To some extent, our findings suggest that CDC5L may serve as a novel therapeutic target for human colorectal cancer.

\section{Materials and Methods}

Cell culture and chemicals

The human colon cancer cell lines DLD1, LoVo, RKO and SW620 were obtained from the American Type Culture Collection (ATCC, Manassas, VA, USA). The SW620 and RKO cells were cultured in Dulbecco's 


\section{Cellular Physiology Cell Physiol Biochem 2017;41:2475-2488

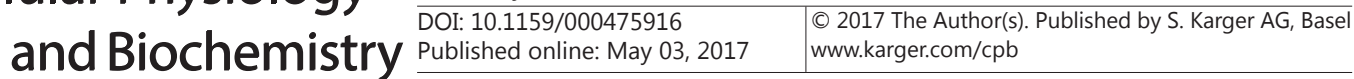

Li et al.: CDC5L Promotes hTERT Expression

modified Eagle's medium (HyClone, Logan, UT, USA). The LoVo and DLD1 cells were cultured in Roswell Park Memorial Institute (RPMI) 1640 medium (HyClone, Logan, UT, USA). All of the growth media were supplemented with 10\% fetal bovine serum (FBS) (HyClone, Logan, UT, USA), and the cells were maintained in a humidified atmosphere with $5 \% \mathrm{CO}_{2}$ at $37^{\circ} \mathrm{C}$.

\section{Nuclear extraction}

The cells were lysed in $250 \mu \mathrm{l}$ of cytoplasmic lysis buffer (10 mM Hepes, pH 7.9, $10 \mathrm{mM} \mathrm{KCl,} 1.5 \mathrm{mM}$ $\mathrm{MgCl}_{2} \cdot 6 \mathrm{H}_{2} \mathrm{O}, 0.5 \% \mathrm{NP}-40$, and $300 \mathrm{mM}$ sucrose) with multiple protease inhibitors ( $1 \mathrm{mM} \mathrm{Na}_{3} \mathrm{VO}_{4}, 10 \mathrm{mM}$ $\mathrm{NaF}, 2.5 \mathrm{mM} \beta$-glycerophosphate, $0.1 \mathrm{mM}$ PMSF, $1 \mathrm{~g} / \mathrm{ml}$ leupeptin, and $0.5 \mathrm{mM}$ dithiothreitol) on ice for 10 min. The mixture was vortexed briefly and centrifuged at $2600 \times \mathrm{g}$ for $1 \mathrm{~min}$ at $4^{\circ} \mathrm{C}$. The supernatant was removed to a new tube and stored at $-80^{\circ} \mathrm{C}$. The pellet was resuspended with $70-100 \mu \mathrm{l}$ of nucleic acid lysis buffer (20 mM Hepes, pH 7.9, $420 \mathrm{mM} \mathrm{NaCl}, 1.5 \mathrm{mM} \mathrm{MgCl} \cdot 6 \mathrm{H}_{2} \mathrm{O}, 0.1 \mathrm{mM}$ EDTA, and 2.5\% glycerol) with multiple protease inhibitors and kept on ice for $30 \mathrm{~min}$. Nuclear proteins were extracted by centrifuging at $10,400 \times \mathrm{g}$ for $10 \mathrm{~min}$ at $4^{\circ} \mathrm{C}$; the resulting supernatant was the nuclear extract. The protein concentrations were determined using the BCA assay.

\section{Streptavidin-agarose pulldown assay}

Biotin-labeled double-stranded oligonucleotide probes corresponding to the -378/-159 fragments of the hTERT promoter sequence were synthesized by TaKaRa Company (sense, 5'-ACC CTG GGA GCG CGA GCG GC-3' and antisense, 5'-GGG GCG GGG TCC GCG CGG AG-3'). The assay was performed by mixing 400 $\mu \mathrm{g}$ of nuclear proteins from different cell lines, $4 \mu \mathrm{g}$ of the double-stranded biotinylated probe and $50 \mu \mathrm{l}$ of the streptavidin-agarose bead solution (Sigma), followed by incubation on a rotating wheel at room temperature for $2 \mathrm{~h}$. The beads were pelleted by centrifugation at $600 \times \mathrm{g}$ for $1 \mathrm{~min}$ and then washed 3 times with $200 \mu \mathrm{l}$ of PBSI. The collected beads were resuspended with $30 \mu \mathrm{l}$ of loading buffer and boiled at $100^{\circ} \mathrm{C}$ for $5 \mathrm{~min}$. The supernatant containing the bound proteins was separated by SDS-PAGE for analysis.

\section{Identification of hTERT promoter-binding proteins}

The hTERT promoter-binding proteins were separated by 10\% SDS-PAGE and visualized by silver staining according to the suggested protocol (Beyotime, China). The protein bands of interest in the gel were cut and digested with trypsin. Identification of the digested samples was performed through mass spectrometry analysis. The proteins indicated by the bands of interest were further identified by searching the available proteomics databases.

\section{Chromatin immunoprecipitation assay (ChIP)}

A $1 \%$ formaldehyde solution was added to the cell culture medium for 10 min to induce cross-linking, and $0.125 \mathrm{M}$ glycine was added to stop the cross-linking. The cells were rinsed twice with ice-cold phosphate buffered saline (PBS), scraped, and collected by centrifugation. The cells were resuspended with $500 \mu \mathrm{l}$ of IP Buffer (100 mM NaCl, 5 mM EDTA, 10 mM Tris-HCl, pH 8.0, 0.02\% NaN3, 10\% SDS, and 5.0\% Triton X-100) for three rounds of sonication of $20 \mathrm{~s}$ per round, and the debris was removed by centrifugation. Then, 50 $\mu \mathrm{l}$ of lysate was used as the DNA input control, and the remaining lysate was incubated with an anti-CDC5L antibody or non-immune rabbit IgG overnight at $4^{\circ} \mathrm{C}$. Immunoprecipitated complexes were collected using protein A/G agarose beads and extensively washed with Micelle Wash Buffer, Buffer500, LiCl detergent solution, and TE Buffer. Then, 1\% SDS and $0.1 \mathrm{M} \mathrm{NaHCO3}$ were added to the immunoprecipitates, which were incubated at $65^{\circ} \mathrm{C}$ overnight and then treated with $400 \mu \mathrm{g} / \mathrm{ml}$ of proteinase $\mathrm{K}$ for $2 \mathrm{~h}$ at $37^{\circ} \mathrm{C}$. DNA was extracted with phenol/chloroform and precipitated with ethanol. The pellets were resuspended in $100 \mu \mathrm{l}$ of ddH2O and subjected to PCR amplification using primers specific for the hTERT promoter (sense 5'-ACC CTG GGA GCG CGA GCG GC and antisense 5'-, GGG GCG GGG TCC GCG CGG AG). The resulting 220-bp product for the hTERT promoter was separated by $1 \%$ agarose gel electrophoresis.

Analysis of hTERT promoter activity

Cells (200,000 cells/well) plated in six-well plates were transfected with hTERT promoter luciferase plasmids encapsulated with DC-nanoparticles. The cells were co-transfected with either a CDC5L-specific siRNA or a negative control siRNA. Forty-eight h after treatment, the expressed luciferase activity was measured as described using a luciferase reporter assay kit (BioVision, Inc., CA, USA). The OD was calculated to correct for variation in the transfection efficiency. 
SiRNA design and transfection

The siRNAs targeting CDC5L (siRNA1: 5'-GGA UGA ACU UGA GAU GCU UTT- 3' and 5'-AAG CAU CUC AAG UUC AUC CTT-3'; siRNA2: 5'-GGA AGA AGC AAG ACG UCU UTT-3' and 5'-AAG ACG UCU UGC UUC UUC CTT-3'; and SiRNA3: 5' GCG UGU AAA GGA AAU GAA ATT-3' and 5'- UUU CAU UUC CUU UAC ACG CTT-3') and a negative control siRNA (5'-UUC UCC GAA CGU GUC ACG UTT-3' and 5'-ACG UGA CAC GUU CGG AGA ATT$3^{\prime}$ ) were purchased from Shanghai GenePharma Co. (Shanghai, China). Cells were plated in 96-well plates (3000 cells/well) or six-well plates (200,000 cells/well) and transfected with siRNA duplexes $(1-2 \mu \mathrm{g} / \mathrm{ml}$ ) encapsulated with DC-nanoparticles. At $48 \mathrm{~h}$ after treatment, protein expression and cell viability were tested by Western blotting and MTT analysis, respectively.

\section{Transient transfection}

siRNAs were purchased from Shanghai GenePharma Co. (Shanghai, China). A total of $2 \times 105$ LoVo cells were seeded into each well of a six-well tissue culture plate (Costar). The next day (when the cells were 70$80 \%$ confluent), the culture medium was aspirated, and the cell monolayer was washed with pre-warmed sterile PBS. The control siRNA was transfected into the LoVo cell lines according to the Lipofectamine 2000 protocol (Invitrogen, USA). The cells were harvested after $48 \mathrm{~h}$ of transfection, and Western blotting or other experiments were performed.

\section{Western blotting analysis}

Cell lysates were separated by electrophoresis in a $10 \%$ sodium dodecyl sulfate-polyacrylamide gradient minigel (SDS-PAGE) and electrophoretically transferred to a polyvinylidene difluoride (PVDF) membrane. The Western blots were probed with specific antibodies against hTERT, CDC5L (Abcam), phospho-AKT, AKT (CST), GAPDH, and $\beta$-actin (Proteintech). The protein bands were detected by electrochemiluminscence (ECL).

\section{Immunofluorescence and confocal microscopy}

Cells were seeded onto coverslips in a 6-well plate, fixed with 4\% paraformaldehyde (w/v) for 30 min, washed for 10 min with PBS and then permeabilized with $0.2 \%$ (w/v) Triton X-100 in PBS for 5 min. The blocking step was performed for 30 min in PBS containing $1 \%$ bovine serum albumin (BSA). The cells were incubated overnight with a primary CDC5L antibody diluted in PBS containing 10\% BSA. After washing with PBS, the cells were incubated for $1 \mathrm{~h}$ with a secondary fluorescein isothiocyanate (FITC) or tetramethylrhodamine isothiocyanate (TRITC)-conjugated antibody. After several additional washing steps, the nuclei were stained with 4',6-diamidino-2-phenylindole (DAPI), and the localization of the CDC5L protein was assessed using a Leica DM 14000B confocal microscope.

\section{Cell viability assay}

Cell viability was determined by the MTT assay (Roche Diagnosis, Indianapolis, IN, USA). Briefly, cells plated in 96-well plates (3000 cells/well) were treated with a siCDC5L or the NSP siRNA. Cell viability was determined $48 \mathrm{~h}$ after treatment.

Clone formation assay

Cells plated in 6-well plates were transfected with a siCDC5L or NSP siRNA. After $24 \mathrm{~h}$, the cells were washed with PBS and trypsinized. Then, the cells were seeded at a density of 100 cells/well in 6-well plates and dispersed evenly by slightly shaking the dishes. The plates were incubated at $37^{\circ} \mathrm{C}$ with $5 \% \mathrm{CO} 2$ for 14 days. The medium was discarded, and the cells were carefully washed twice with PBS. After fixation with $4 \%$ paraformaldehyde for $15 \mathrm{~min}$, the cells were stained with $0.1 \%$ crystal violet for $15 \mathrm{~min}$, washed with tap water and air dried. Clones with more than 50 cells were counted with an optical microscope. The clone formation rate was calculated using the following formula: plate clone formation inhibitory ratio=(number of clones treated with siRNA/number of cells inoculated) $\times 100 \%$.

Scratch assay

A scratch assay was performed to detect cell migration. The cells were grown to full confluence in six-well plates and incubated overnight. The cell monolayers were wounded with a sterile $200 \mathrm{ml}$ pipette tip and washed with PBS to remove detached cells from the plates. The cells were treated with a siCDC5L 


\section{Cellular Physiology Cell Physiol Biochem 2017;41:2475-2488 \\ \begin{tabular}{l|l} 
and Biochemistry Published 10.1159/000475916 2017 & $\begin{array}{l}\text { ( ) 2017 The Author(s). Published by S. Karger AG, Basel } \\
\text { www.karger.com/cpb }\end{array}$
\end{tabular}}

Li et al.: CDC5L Promotes hTERT Expression

or NSP siRNA and incubated in a CO2 incubator. After $72 \mathrm{~h}$, the medium was replaced with PBS buffer, the wound gap was observed, and the cells were photographed using an Olympus microscope fitted with a digital camera.

\section{Immunohistochemistry analysis}

The 130 colorectal cancer samples and adjacent non-carcinoma tissues used for immunostaining analysis of CDC5L and hTERT protein expression were collected at the Liaoning Cancer Hospital. These tissue samples were previously obtained with informed consent from patients who had not undergone anti-cancer treatment prior to tumor resection. Detailed clinical and pathological information, including the clinical and pathologic TNM stage and OS duration, was available for all cases. The tissue specimens were histologically examined and classified according to the 2004 World Health Organization classification system (Travis WD: Pathology of pulmonary vasculitis. Sem Resp Crit Care M 2004, 25:475-482). The pathological TNM status of all colorectal cancers was assessed according to the criteria of the seventh edition of the American Joint Committee on Cancer (2010).

Immunohistochemistry was conducted using the Envisionp Kit/HRP (DakoCytomation). Briefly, slides were immersed in Target Retrieval Solution ( $\mathrm{pH}$ 9; DakoCytomation) and boiled at $108^{\circ} \mathrm{C}$ for $15 \mathrm{~min}$ in an autoclave for antigen retrieval. An anti-CDC5L or anti-hTERT antibody was added to each slide after blocking endogenous peroxidase and proteins, and the sections were incubated with HRP-labeled anti-rabbit IgG as the secondary antibody. The chromogen substrate was added, and the specimens were counterstained with hematoxylin. A negative control was obtained by replacing the primary antibody with normal rabbit IgG.

The degrees of immunostaining were reviewed and scored by two independent observers using light microscopy (magnification 200x). Cytoplasm that stained brown was positive. The staining intensity was graded as follows: absent staining as “-”, weak as “+", moderate as “++", and strong as "+++." Because each tissue on the tissue microarray slides was quite small, we could not determine the percentages of stained cells. Stained tissues with "++" and "+++" were determined to have high CDC5L and hTERT expression, whereas tissues with "-" and "+" were judged to have low CDC5L and hTERT expression. The results were provided by two senior pathologists.

\section{Acquisition of carcinoma tissue samples}

Colorectal cancer samples and adjacent non-carcinoma tissuesfrom colorectal carcinoma patients with different histological types $(n=6)$ from the First Affiliated Hospital of Dalian Medical University (Dalian, China) were used. All of the samples were stored in liquid nitrogen prior to the Western blotting analysis. Informed consent was obtained from each patient, and the entire study was approved by the Committees on Human Rights in Research at Dalian Medical University.

\section{Xenograft mouse model and evaluation of angiogenesis factors in xenograft tumor tissues}

The animal experiments were performed in accordance with the National Institutes of Health Guide for the Care and Use of Laboratory Animals under the approval of the specific pathogen-free (SPF) Laboratory Animal Center at Dalian Medical University. The cholesterol-conjugated CDC5L siRNA and negative control siRNA for in vivo delivery were obtained from Shanghai GenePharma Co. (Shanghai, China). The knockdown efficiency of these siRNAs was validated in vitro. To investigate the effect of CDC5L inhibition on colon cancer cell growth in vivo, LoVo cells $(5 \times 106$ cells $/ \mathrm{mL})$ were inoculated subcutaneously into the flanks of nude mice. Once palpable tumors were observed, tumor volume measurements were recorded every two days using calipers. The mice were randomly divided into the following 2 groups ( 6 mice per group) after the tumor volume reached nearly $100 \mathrm{~mm}$ in size: (a) control siRNA and (b) CDC5L siRNA. For delivery of DC nanoparticle-conjugated siRNA, $10 \mu \mathrm{g}$ of siRNA in $0.1 \mathrm{ml}$ of saline buffer was injected intratumorally three times a week for 3 weeks. The tumor volume was calculated as $V=($ width $2 \times$ length $) / 2$ and was measured using digital calipers. Finally, the mice were sacrificed, and the tumor size and weight were photographed and recorded, respectively. Tumor specimens were fixed in formalin and embedded in paraffin for CDC5L, P-AKT,AKT and hTERT protein expression analysis. Immunohistochemical staining was performed as described below. One part of the sample was used for protein extraction and further analyzed by Western blotting. The remainder of the sample was embedded for histological analysis. A negative control was obtained by replacing the primary CDC5L or hTERT antibody with normal rabbit or mouse IgG. The positive immunoreactive cells from each of the differently treated tumor tissue sections were measured at $200 \times$ 
magnification using a light microscope. The amount of protein was analyzed by integral optical density (IOD) using the IPP software (Image-ProPlus 6.0, Bethesda, MD, USA).

\section{Statistical analysis}

Analysis of variance and Student's t test were used to compare the test and control sample values. $\mathrm{P}<0.05$ was considered a significant difference. The SPSS 21.0 software was used for all statistical analyses. Significance was evaluated using the paired $t$ test. All experiments were performed three times, and the mean \pm SEM was calculated.

\section{Results}

Discovery and validation of hTERT promoter-binding proteins in colon cancer cells

To discover novel and specific hTERT promoter regulators in colon cancer cell lines, we designed and synthesized a220-bp biotin-labeled double-stranded oligo nucleotide probe corresponding to the $5^{\prime}$-flanking sequence of the hTERT gene (from -378 bp to -159 bp) as a DNA probe to pull down transcription factors from the hTERT gene and to assess their binding to the hTERT promoter region. Nuclear extracts prepared from human colon cancer cell lines (SW620, RKO, DLD1 and LoVo) were incubated with the biotin-labeled hTERT promoter probe and streptavidin-agarose beads. The pulled down and purified protein factors that bound to the hTERT promoter probe were separated by SDS-PAGE and then visualized by silver staining (Fig. 1A). Based on their differential binding capabilities to the hTERT promoters of the four colon cancer cell lines, we selected protein bands of interests for further analysis using mass spectrometry and proteomic techniques. One protein band with a molecular weight of approximately $100 \mathrm{kDa}$ was identified as CDC5L.

To verify the binding of CDC5L to the hTERT promoter region in colon cancer cells, we first analyzed the binding of CDC5L to the chromatin hTERT promoter in living cells using the chromatin immunoprecipitation (ChIP) assay with a specific antibody against CDC5L. Normal IgG was used as the negative control. Then, DNA immunoprecipitated from the colon cancer cell lines (SW620, RKO, DLD1 and LoVo) by an antibody against CDC5L was amplified by PCR using primers corresponding to the hTERT promoter region (-378 bp to $-159 \mathrm{bp})$. The hTERT promoter was amplified from the colon cancer cells, which verified the binding of the CDC5L protein to the endogenous hTERT promoter. We did not detect amplification

A

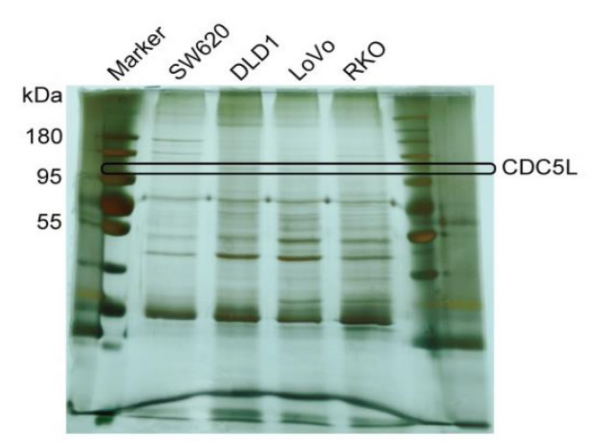

B

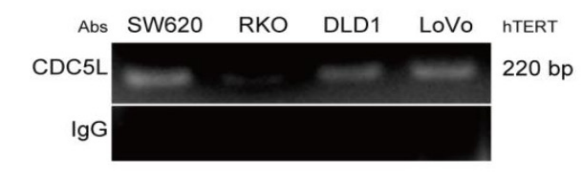

C

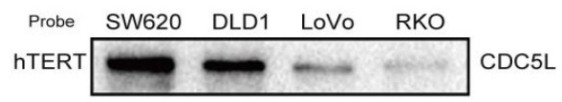

Fig. 1. CDC5L was identified and validated as a specific binding protein of the hTERT promoter in colon cancer cells. (A) A streptavidin-biotin pulldown assay was performed to determine the specific proteins that bound to the hTERT promoter. Nuclear extracts prepared from human colon cancer cell lines (SW620, DLD1, LoVo and RKO) were incubated with a biotin-labeled hTERT promoter probe (-378 to-159 bp) and streptavidin-agarose beads. The DNA-protein complexes were separated by SDS-PAGE, and the protein bands were visualized by silver staining. The box indicates the candidate hTERT promoter-binding protein. (B) ChIP assays were performed using the hTERT promoter from colon cancer cells. PCR products were separated on $1 \%$ agarose gels. Normal IgG was included as a negative control. (C) Immunoblotting assay to detect CDC5L binding to the hTERT promoter probe (-378 to $-159 \mathrm{bp}$ ). The CDC5L protein in the DNA-protein complexes was detected by Western blotting using an anti-CDC5L antibody. 


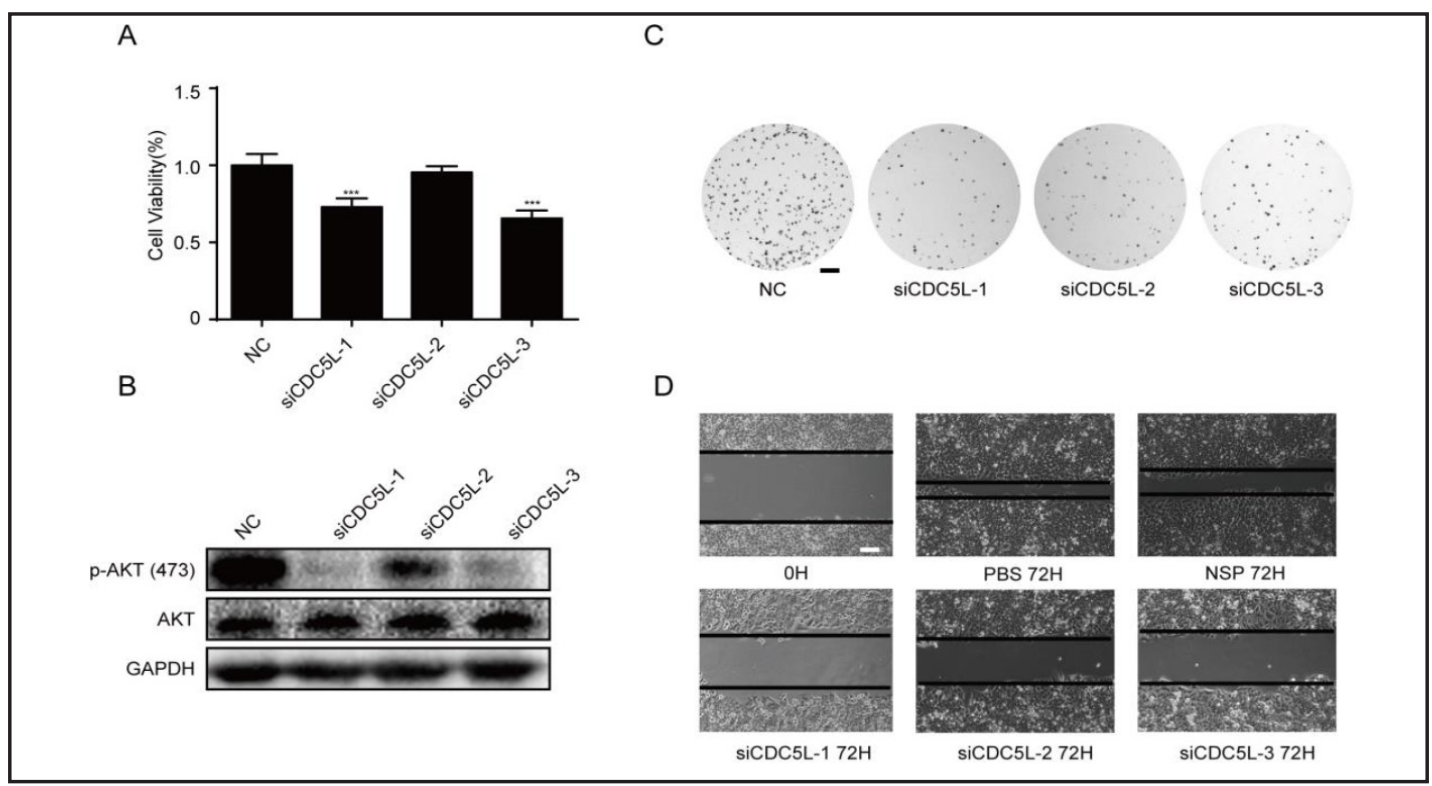

Fig. 2. CDC5L regulated colon cancer cell growth. Cell viability was analyzed using the MTT assay in LoVo cells transfected with the siCDC5L or nonspecific control siRNA for $48 \mathrm{~h}$. (B) AKT and its phosphorylated form in LoVo cells transfected with siCDC5L were detected by Western blotting using antibodies against AKT and p-AKT, respectively. (C) A colony formation assay was performed in LoVo cells transfected with a siCDC5L or nonspecific control siRNA twice a week for two weeks. The quantification of colonies is shown. (D) Cell migration was analyzed using a wound-healing assay. LoVo cells were seeded into 6-well plates and grown to full confluence. Cell migration was measured as described (50x). The data are presented as the mean \pm SEM of three separate experiments. $(* * * P<0.001)$.

of the hTERT promoter when the negative IgG control was used in the ChIP assay (Fig. 1B).

To verify the binding of CDC5L to the hTERT promoter region in the chromatin structure in living cells, we performed a Western blotting assay to detect the proteins eluted from the biotin-streptavidin pulldown complexes using an anti-CDC5L antibody. As shown in Fig. 1C, all four colon cancer cell lines (SW620, RKO, DLD1 and LoVo) demonstrated tumor cellselective binding of CDC5L to the hTERT promoter. These results demonstrated that CDC5L specifically bound to the hTERT promoter in human colon cancer cells.

\section{Effect of CDC5L regulation on colon cancer cell proliferation and migration}

The results above indicated that hTERT was regulated by CDC5L. Next, we analyzed the effect of CDC5L on LoVo cell proliferation using the MTT assay. As shown in Fig. 2A, inhibition of CDC5L expression with siRNAs targeting CDC5L (siCDC5L) dramatically suppressed LoVo cell proliferation, especially siCDC5l-1 and siCDC5L-3.

We then examined expression of PI3K/AKT family protein members and verified the role of CDC5L in activating PI3K/AKT proteins. As shown in Fig. 2B, AKT phosphorylation was decreased by CDC5L knockdown, although the total phosphorylation level did not change. From the results above, we concluded that CDC5L was specifically expressed in colon cancer cells and was correlated with cell growth and proliferation partially through regulation of the PI3K/AKT signaling pathway.

We also tested the effects of CDC5L on tumor cell clonogenicity in LoVo cells (Fig. 2C). Treatment with siCDC5L markedly inhibited colony formation, indicating a lower colony formation activity than that of the controls.

Additionally, CDC5L knockdown significantly inhibited cell migration. As shown in Fig. 2D, the wounded space between the cell layers from a scratch was completely occupied by the migrating cells after $72 \mathrm{~h}$ in the control group. However, this space was not occupied by migrating cells treated with siCDC5L.

\section{KARGER}


A

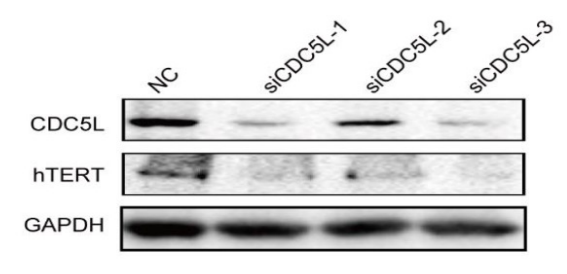

B

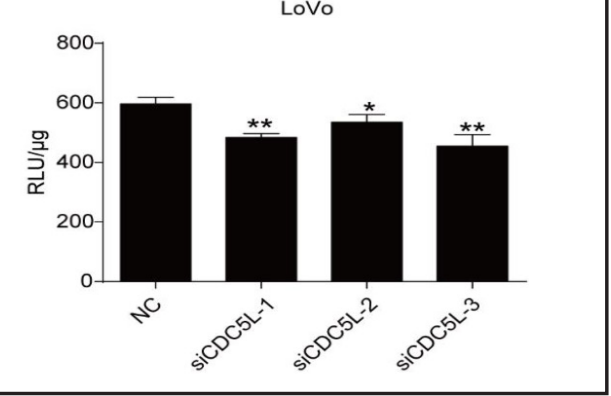

Fig. 3. CDC5L promoted the transcriptional activation of the hTERT promoter. (A) Down-regulation of hTERT protein expression by CDC5L knockdown. LoVo cells were transfected with a CDC5L siRNA or nonspecific control siRNA for $48 \mathrm{~h}$, and hTERT protein expression was analyzed by Western blotting. (B) LoVo cells were co-transfected with the siCDC5L and hTERT promoter-driven luciferase (-378/-159 bp) plasmids. Luciferase activity was detected as described previously.

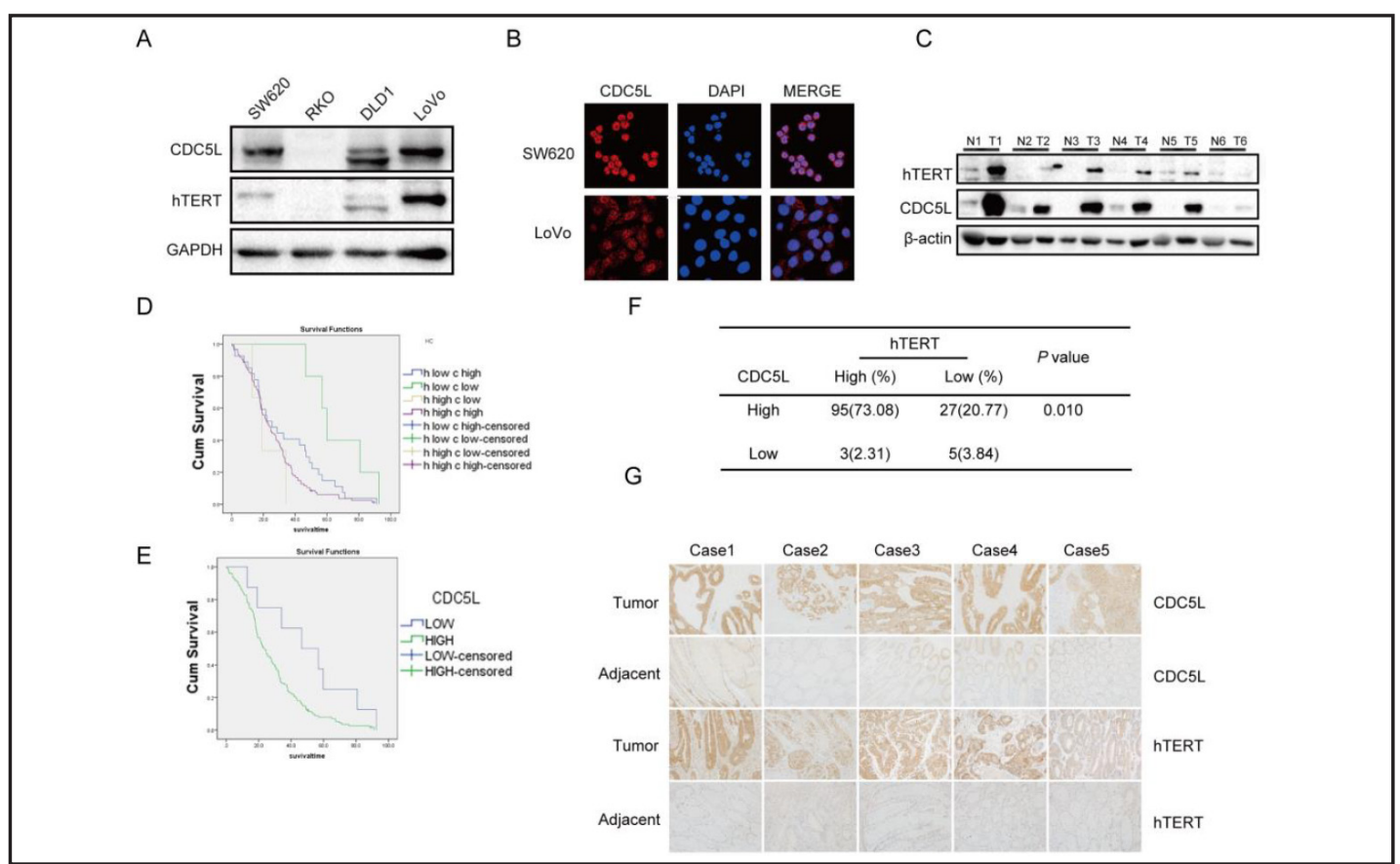

Fig. 4. Analysis of CDC5L and hTERT protein expression from a human colorectal cancer tissue microarray. (A) CDC5L and hTERT expression in colon cancer cells (the SW620, DLD1, LoVo and RKO cell lines) was detected by Western blotting using anti-CDC5L and hTERT antibodies. (B) CDC5L expression in colon cancer cells (SW620 and LoVo) was detected by immunofluorescence. The localization of CDC5L is shown. Red staining by secondary tetramethylrhodamine isothiocyanate-conjugated antibodies indicatesCDC5L, and blue staining by DAPI indicates the nucleus. (C) The protein samples extracted from six human colorectal carcinoma tissues and adjacent tissues were used to examine CDC5L and hTERT expression. (D) Kaplan-Meier analysis of OS with high or low CDC5L and hTERT expression $(P<0.001$, log-rank test). (E) Kaplan-Meier analysis of OS with high or low CDC5L expression $(P<0.001$, log-rank test). (F) The correlation between CDC5L and hTERT expression in colorectal cancer tissues from 130 patients is shown. (G) High CDC5L and hTERT expression was indicated by brown staining, and low or no expression was shown by lighter staining. Cases 1-5 are five representative cases from five different patients. The tumor and adjacent tissues were collected from one patient for each case.

CDC5L promoted the transcriptional activation of the hTERT promoter

We evaluated the effect of CDC5L on hTERT expression at the protein level. hTERT protein expression was down-regulated by CDC5L knockdown. LoVo cells were transfected 
with a CDC5L siRNA or a negative control siRNA for $48 \mathrm{~h}$, and hTERT protein expression was analyzed by Western blotting. Knockdown of CDC5L by the CDC5L siRNA significantly decreased the expression of hTERT in the LoVo cells (Fig. 3A).

Based on the results described above, CDC5L may play a crucial role in the regulation of hTERT transcription. To verify this hypothesis, we constructed a luciferase reporter vector driven by the hTERT promoter (shown in Fig. 3B). We co-transfected the luciferase reporter plasmid driven by the hTERT promoter $(-378 \mathrm{bp} /-159 \mathrm{bp})$ and a siCDC5L into LoVo cells, which had high CDC5L expression. At $48 \mathrm{~h}$ after treatment, the inhibition of CDC5L expression by siCDC5L-1, siCDC5L-2 or siCDC5L-3 attenuated hTERT luciferase activity (-378 bp/-159 bp) compared with the negative control group (Fig. 3B). These results indicate that CDC5L regulates the transcriptional activation of hTERT.

Overexpression of CDC5L and hTERT in colorectal carcinoma cells and tumor tissues and their association with patient survival

To confirm the correlation between CDC5L and hTERT and their biological and clinicopathologic significance in colorectal cancer patients, we evaluated CDC5L and hTERT expression in colorectal cancer cells by Western blotting. As shown in Fig. 4A, CDC5L was highly expressed in the colon cancer cell lines SW620, DLD1 and LoVo but was expressed at a lower level in the RKO colon cancer cells. hTERT showed almost the same expression trend as CDC5L in these cell lines. Immunofluorescence analysis was used to analyze the expression and sub-cellular localization of CDC5L in colon cancer cells, and CDC5L was found to be highly expressed in the nuclei in the colon cancer LoVo and SW620 cell lines (Fig. 4B).

We next assessed CDC5L and hTERT expression in colorectal cancer tissues and corresponding adjacent non-cancer tissues by Western blotting. Both CDC5L and hTERT were highly expressed in the colorectal cancer tissues compared with the adjacent noncancer tissues (Fig. 4C).

Detailed clinical and pathological information, including the clinical and pathological tumor-node-metastasis (TNM) stage and overall survival (OS) duration, was available for all 130 cases. Thus, we analyzed the synergistic effect of CDC5L and hTERT expression on the survival rates of patients with colorectal cancer using Kaplan-Meier analysis. The OS curves indicated that patients with low hTERT and CDC5L expression had significantly higher survival rates than patients with high expression of both proteins $(P<0.01$, Fig. 4D). The survival rates of the patients were significantly correlated with the CDC5L expression level (Fig. 4E). Among the 130 patient tumor tissue samples, approximately 95 (73.08\%) patients showed high expression of both hTERT and CDC5L, whereas only $5(3.84 \%)$ cases showed low expression of both hTERT and CDC5L. Furthermore, Pearson correlation analysis showed that CDC5L expression was positively correlated with hTERT expression $(P<0.01)$ (Fig. 4F). Similarly, immunohistochemical staining showed that colorectal tumor tissues but not adjacent non-cancer tissues had high CDC5L and hTERT expression. Five representative cases (Cases 1-5) selected from five different patients are shown (Fig. 4G).

The prognosis of colorectal cancer patients was not closely associated with the gender, age, location, tumor size, or histological type but was significantly associated with the differentiation, $\mathrm{N}$ stage, TNM stage and the correlation between CDC5L and hTERT expression (Table 1). The results demonstrated a potential correlation between the CDC5L and hTERT expression levels and their indication for a poor prognosis in colorectal cancer patients. Multivariate analysis using the Cox's proportional hazards model revealed that CDC5L, differentiation, and the $\mathrm{N}$ stage were independent prognostic indicators for OS in colorectal patients $(P<0.05$; Table 2, 3).

These findings indicated that CDC5L was an independent prognostic factor for the OS of colorectal patients.

Inhibition of tumor growth by CDC5L knockdown through down-regulation of hTERT expression in mice

The effect of CDC5L on tumor growth was examined in nude mice with human colon tumor xenografts. Human colon cancer cells (LoVo) were injected subcutaneously into the 
armpits of nude mice. When the tumors were nearly $100 \mathrm{~mm}^{3}$ in size, the animals were treated with a CDC5L-specific siRNA and a nonspecific (NSP) control siRNA encapsulated by DC-nanoparticles. The tumor volumes and weights from the mice treated with a CDC5L-specific siRNA were smaller and lighter than the values obtained for the group treated with the nonspecific RNA (Fig. 5A, B, D). Furthermore, we examined the effect of CDC5L knockdown on hTERT expression and AKT phosphorylation at the protein level in the xenografts using Western blotting and immunohistochemistry analyses. As shown in Fig. 5C and 5E, CDC5L-specific siRNA treatment attenuated hTERT and p-AKT expression in the xenografts. The above results have demonstrated the important role of CDC5L in colorectal tumor growth, partially through the regulation of hTERT expression.

\section{Discussion}

CRC is the third leading cause of cancerrelated death worldwide because the survival rate remains low [31]. Currently, studies are investigating the etiology, pathogenesis and epidemiology of CRC; however, their molecular mechanisms remain poorly understood. Classical chemical drugs that perturb mitotic progression have been successful in the clinical treatment of cancer, but side effects limit their use. These circumstances
Table 1. Kaplan-meier analyses for prognosis of 130 CRC patients

\begin{tabular}{|c|c|c|c|c|c|c|c|}
\hline & \multirow{2}{*}{$\begin{array}{c}\text { frequenc } \\
y\end{array}$} & \multirow{2}{*}{ MST } & \multicolumn{2}{|c|}{$95 \% \mathrm{CI}$} & \multirow{2}{*}{$\begin{array}{c}\text { Survival Rate } \\
5-y\end{array}$} & \multirow{2}{*}{$\chi^{2}$} & \multirow{2}{*}{$P$} \\
\hline & & & lower & upper & & & \\
\hline \multicolumn{8}{|l|}{ gender } \\
\hline male & 70 & $27.700 \pm 5.491$ & 16.938 & 38.462 & $15.5 \%$ & 2.715 & 0.099 \\
\hline female & 60 & $22.100 \pm 3.357$ & 15.521 & 28.679 & $1.7 \%$ & & \\
\hline \multicolumn{8}{|l|}{ age } \\
\hline$\leq 60$ & 57 & $21.900 \pm 3.397$ & 15.242 & 28.558 & $9.9 \%$ & 0.039 & 0.844 \\
\hline$>60$ & 73 & $24.700 \pm 3.085$ & 18.653 & 30.747 & $8.2 \%$ & & \\
\hline \multicolumn{8}{|l|}{ location } \\
\hline colon & 41 & $19.200 \pm 1.984$ & 15.311 & 23.089 & $7.3 \%$ & 2.223 & 0.136 \\
\hline recta & 89 & $27.900 \pm 3.249$ & 21.531 & 34.269 & $9.7 \%$ & & \\
\hline \multicolumn{8}{|l|}{ size } \\
\hline$\leq 5 \mathrm{~cm}$ & 78 & $27.000 \pm 4.637$ & 17.912 & 36.088 & $11.2 \%$ & 2.574 & 0.109 \\
\hline$>5 \mathrm{~cm}$ & 52 & $20.800 \pm 3.906$ & 13.144 & 28.456 & $5.8 \%$ & & \\
\hline \multicolumn{8}{|l|}{ Histologicaltype } \\
\hline adenocarcinoma & 113 & $25.500 \pm 2.862$ & 19.891 & 31.109 & $9.4 \%$ & 1.346 & 0.246 \\
\hline other & 17 & $19.200 \pm 1.543$ & 16.175 & 22.225 & $5.9 \%$ & & \\
\hline \multicolumn{8}{|l|}{ differentiation } \\
\hline high & 48 & $32.300 \pm 1.732$ & 28.905 & 35.695 & $16.7 \%$ & 10.478 & 0.005 \\
\hline moderate & 70 & $20.800 \pm 2.510$ & 15.880 & 25.720 & $3.8 \%$ & & \\
\hline low & 12 & $12.500 \pm 3.294$ & 6.050 & 18.950 & $0.0 \%$ & & \\
\hline \multicolumn{8}{|l|}{ infiltration } \\
\hline serosa & 94 & $25.400 \pm 3.151$ & 19.224 & 31.576 & $9.1 \%$ & 1.846 & 0.174 \\
\hline beyondserosa & 36 & $20.800 \pm 3.300$ & 14.332 & 27.268 & $8.3 \%$ & & \\
\hline \multicolumn{8}{|l|}{ Nstage } \\
\hline N0 & 46 & $34.100 \pm 8.026$ & 18.370 & 49.830 & $19.0 \%$ & 12.379 & 0.002 \\
\hline N1 & 48 & $21.400 \pm 2.540$ & 16.421 & 26.379 & $6.3 \%$ & & \\
\hline N2 & 36 & $24.600 \pm 4.950$ & 14.898 & 34.302 & $0.0 \%$ & & \\
\hline \multicolumn{8}{|l|}{ TNM } \\
\hline stageII & 46 & $34.100 \pm 8.026$ & 18.370 & 49.830 & $19.0 \%$ & 11.596 & 0.001 \\
\hline stagellI & 84 & $21.900 \pm 2.658$ & 16.691 & 27.109 & $3.6 \%$ & & \\
\hline \multicolumn{8}{|l|}{ hTERT } \\
\hline LOW & 32 & $32.700 \pm 14.637$ & 4.011 & 61.389 & $18.8 \%$ & 7.840 & 0.005 \\
\hline $\mathrm{HIGH}$ & 98 & $22.500 \pm 2.623$ & 17.358 & 27.642 & $2.4 \%$ & & \\
\hline \multicolumn{8}{|l|}{ CDC5L } \\
\hline LOW & 8 & $46.500 \pm 16.051$ & 15.039 & 77.961 & $25.0 \%$ & 5.625 & 0.018 \\
\hline $\mathrm{HIGH}$ & 122 & $23.000 \pm 2.798$ & 17.516 & 28.484 & $7.9 \%$ & & \\
\hline \multicolumn{8}{|c|}{ 年 } \\
\hline H LOW C HIGH & 27 & $25.400 \pm 6.231$ & 13.187 & 37.613 & $14.8 \%$ & 11.609 & 0.009 \\
\hline H LOW C LOW & 5 & $59.800 \pm 3.286$ & 53.359 & 66.241 & $40.0 \%$ & & \\
\hline H HIGH C LOW & 3 & $19.100 \pm 4.981$ & 9.338 & 28.862 & $0.0 \%$ & & \\
\hline H HIGH C HIGH & 95 & $23.000 \pm 2.702$ & 17.703 & 28.297 & $6.0 \%$ & & \\
\hline
\end{tabular}

Table 2 Categorical Variable Codings ${ }^{\mathrm{a}, \mathrm{c}}$. ${ }^{\mathrm{C} C a t e g o r y}$ variable: differentiation. ' Indicator Parameter Coding. ${ }^{\mathrm{C}}$ Category variable: Nstage

\begin{tabular}{llccc}
\hline & & Frequency & $(1)$ & $(2)$ \\
\hline differentiation $^{\mathrm{b}}$ & 1=high & 48 & 0 & 0 \\
& $2=$ =moderate & 70 & 1 & 0 \\
& $3=$ low & 12 & 0 & 1 \\
& $0=\mathrm{N} 0$ & 46 & 0 & 0 \\
Nstage $^{\mathrm{b}}$ & $1=\mathrm{N} 1$ & 48 & 1 & 0 \\
& $2=\mathrm{N} 2$ & 36 & 0 & 1 \\
\hline
\end{tabular}

Table 3. Univariate analysis of the contribution of various potential prognostic factors to survival in colorectal specimens. a. Degree of freedom reduced because of constant or linearly dependent covariates. $b$. Constant or Linearly Dependent Covariates TNM $=2+\mathrm{N}$ stage (1) $+\mathrm{N}$ stage (2)

\begin{tabular}{|c|c|c|c|c|c|c|c|c|}
\hline \multicolumn{9}{|c|}{ Variables in the Equation ${ }^{b}$} \\
\hline & \multirow{2}{*}{ B } & \multirow{2}{*}{ SE } & \multirow{2}{*}{ Wald } & \multirow{2}{*}{$\mathrm{df}$} & \multirow{2}{*}{ Sig. } & \multirow{2}{*}{$\operatorname{Exp}(B)$} & \multicolumn{2}{|c|}{$95.0 \%$ CI for $\operatorname{Exp}(B)$} \\
\hline & & & & & & & Lower & Upper \\
\hline differentiation & & & 9.946 & 2 & .007 & & & \\
\hline differentiation(1) & .404 & .205 & 3.876 & 1 & .049 & 1.497 & 1.002 & 2.237 \\
\hline differentiation(2) & 1.048 & .345 & 9.239 & 1 & .002 & 2.852 & 1.451 & 5.607 \\
\hline Nstage & & & 6.994 & 2 & .030 & & & \\
\hline Nstage(1) & .463 & .220 & 4.430 & 1 & .035 & 1.588 & 1.032 & 2.444 \\
\hline Nstage(2) & .609 & .247 & 6.107 & 1 & .013 & 1.839 & 1.134 & 2.982 \\
\hline TNM & & & . & $0^{\mathrm{a}}$ & . & & & \\
\hline CDC5L & 1.047 & .408 & 6.575 & 1 & .010 & 2.849 & 1.280 & 6.341 \\
\hline
\end{tabular}


$A$

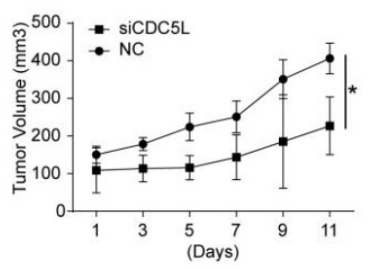

$\mathrm{D}$

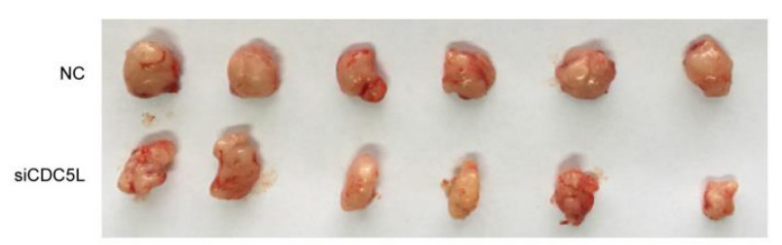

B

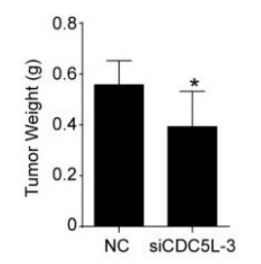

C

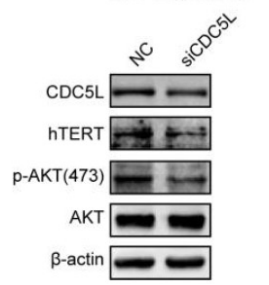

$E$

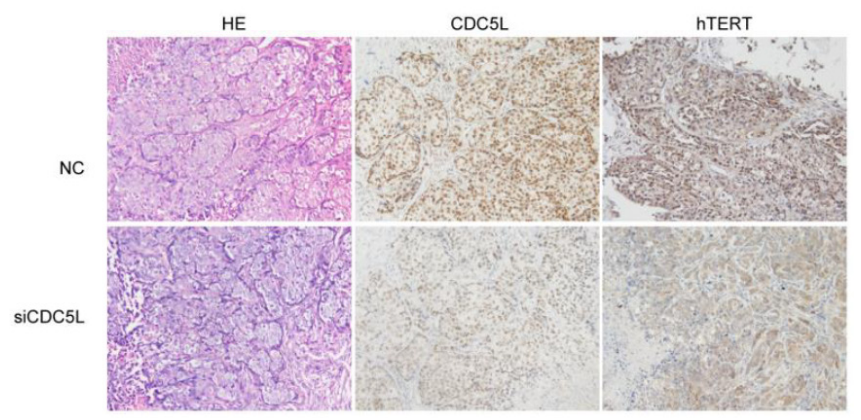

Fig. 5. CDC5L knockdown inhibited tumor growth by down-regulating hTERT expression in a mouse model. (A). The tumor volume of each group of nude mice was measured and calculated as $V=\left(\right.$ width $^{2} \times$ length $/ 2$, $\mathrm{n}=6,{ }^{* *} P<0.01,{ }^{* * *} P<0.001$. (B) The weight of tumor grafts 11 days after the intratumoral injection of PBS or siCDC5L-3, *** $P<0.001$. (C) The proteins were extracted from the tumor xenografts. CDC5L, hTERT, p-AKT, and AKT expression was detected by Western blotting. (D) The morphology of the tumor xenografts from each nude mouse 11 days after treatment. (E) Immunohistochemistry assay to detect CDC5L and hTERT expression from tumor xenografts in each group of nude mice.

have prompted us to search for alternative targets. Here, we found that CDC5L was a promoter-binding protein linked with hTERT in cancer cells via pulldown and ChIP experiments, and we found a correlation between CDC5L and hTERT. Then, we determined that CDC5L was an important factor in CRC cells due to its role as an essential regulator of the p-AKT pathway, as decreased CDC5L expression was shown to directly affect CRC cell proliferation and migration. CDC5L expression in colon cancer cells was then detected by immunofluorescence. These findings have presented a strong correlation between CDC5L and hTERT, which sheds new light on diagnostic and therapeutic techniques for human CRC.

The telomerase subunit hTERT is a core component of the telomerase holoenzyme and is involved in the regulation of telomerase activity [32, 33]. hTERT has been reported to be important for cancer tumorigenesis, growth, migration and invasion [34, 35]. The presence of telomerase in more than $80 \%$ of human cancer cells indicates that the enzyme may be a promising target for cancer therapy. Telomerase inhibitors are likely to kill cancer cells by inducing senescence or apoptosis through telomere shortening, and many different classes of anti-cancer compounds that directly inhibit telomerase have been developed [36]. Most studies have shown that telomeres have unequal lengths in tumors compared with normal tissues [37]. Telomeres determine the genomic stability of a tumor primarily by shortening the telomere lengths in the tumor itself [38]. Previous studies showed that hTERT was a target gene of colorectal cancer [39]. In our study, we found that hTERT was highly expressed in CRC in combination with CDC5L, and the Kaplan-Meier analysis demonstrated a poor clinicopathological outcome associated with this dual expression.

A previous study suggested that CDC5L was highly expressed in some tumors, such as cervical tumors and osteosarcoma. CDC5L was reported to be a component of a pre-mRNA 
splicing factor and to play a crucial role in mitotic progression; downregulation of CDC5L can kill proliferating cancer cells during mitosis. Targeting the mitotic stage of the cell cycle is increasingly considered an effective approach for cancer therapy [40, 41]. Studies have also shown that CDC5L is expressed during cell division, suggesting that its overexpression in mammalian cells may result in the shortening of the G2 phase of the cell cycle [16]. CDC5L regulates the expression levels and splicing efficiencies of a set of genes related to mitosis and DNA damage repair [9]. Moreover, CDC5L is phosphorylated in vivo, which does not affect its nuclear localization or spliceosomal assembly. In our study, we found that AKT phosphorylation was decreased by CDC5L knockdown, although the total phosphorylation level did not change. Thus, we concluded that CDC5L was specifically expressed in colon cancer cells and was correlated with cell growth and proliferation partially through regulating the PI3K/AKT signaling pathway. Manipulation of the phosphorylation sites on CDC5L may provide new strategies to regulate cell division in disorders with unguarded cellular proliferation, such as tumors [42]. Therefore, CDC5L may be an unpredictable factor in tumor pathogenesis. Inhibiting CDC5L has potential as a targeted antimitotic cancer therapy. In our study, inhibiting the expression of CDC5L dramatically suppressed LoVo cell proliferation. Additionally, hTERT expression was correlated with CDC5L expression. However, the function and mechanism of the elevated CDC5L expression level in cancer cells remain unclear [43]. Finally, the results of our evaluation showed that CDC5L targeted CRC cells via the pathogenic mechanisms of hTERT. Inhibiting CDC5L decreased hTERT and tumor growth in animal experiments via the p-AKT pathway.

We confirmed the clinicopathological significance of the correlation between CDC5L and hTERT in colorectal cancer patients, as high CDC5L expression was observed in colorectal cancer cells. The survival rates of patients with low CDC5L expression were significantly higher than the survival rates of patients with high expression of both proteins, indicating that CDC5L was an independent prognostic factor for colorectal patients. We found that CDC5L was significantly related to an advanced TNM stage, which was consistent with previous studies. No significant differences were found for any other clinicopathological parameters, including age, gender, tumor location, tumor size and histological type [44]. These findings indicate that up-regulation of CDC5L may be involved in CRC progression. The synergistic effect of CDC5L and hTERT expression and the survival rates of the patients were significantly correlated with the expression level of CDC5L. Additionally, the CDC5L-specific siRNAs effectively controlled the tumor weight, and the immunohistochemistry analysis showed that CDC5L and hTERT were expressed in tumor xenografts in a nude mouse model.

CDC5L was determined to be highly expressed in CRC cells based on the comparison between the colorectal cancer samples and adjacent non-carcinoma tissues used for the immunostaining analysis of CDC5L and hTERT expression, and high CDC5L expression predicted a poorer outcome in CRC patients. Furthermore, CDC5L exerted its function by directly targeting hTERT. These findings indicate that CDC5L may act as a novel potential therapeutic agent for the treatment of colorectal cancer. This study provides both experimental and theoretical bases for the pathogenesis of CDC5L. In summary, we have identified CDC5L as a novel regulator of hTERT expression with the potential to be a new therapeutic target for CRC.

\section{Acknowlogments}

This work was supported by the funds from the National Natural Science Foundation of China (81173615 XC, 81472178 WD); Science and technique support plane of the first affiliated hospital of Dalian Medical University (2013D005); the State "973 Program" of China (2014CB542005); the Education Department of Liaoning Province in China (the "Program for Pan-Deng Scholars"); and the National Natural Science Foundation of Liaoning Province in China. 


\section{Cellular Physiology Cell Physiol Biochem 2017;41:2475-2488

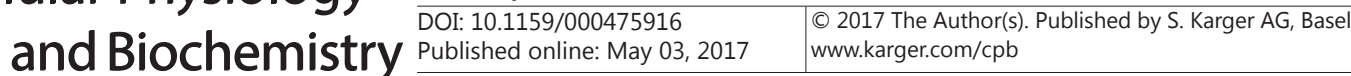

Li et al.: CDC5L Promotes hTERT Expression

\section{Disclosure Statement}

None of the authors listed in this manuscript have a conflict of interest

\section{References}

-1 Chen W, Zheng R, Baade PD, Zhang S, Zeng H, Bray F, Jemal A, Yu XQ He J: Cancer statistics in china, 2015. CA Cancer J Clin 2016;66:115-132.

2 Ning W, Tingting S, Rongshou Z, Siwei Z, Wanqing C: An analysis of incidence and mortality of colorectal cancer in china, 2009. China Cancer 2013;22:515-520.

3 Qiong C, Zhicai L, Lanping C, Guohui S, Xibin S, Rongshou Z, Siwei Z, Wanqing C: An analysis of incidence and mortality of colorectal cancer. China Cancer 2012;21:179-182.

-4 Brenner H, Kloor M, Pox CP: Colorectal cancer. Lancet 2014;383:1490-1502.

-5 Kelly C, Cassidy J: Chemotherapy in metastatic colorectal cancer. Surg Oncol 2007;16:65-70.

-6 Peeters M, Price T, Van Laethem JL: Anti-epidermal growth factor receptor monotherapy in the treatment of metastatic colorectal cancer: where are we today? Oncologist 2009;14:29-39.

7 Jackson JR, Patrick DR, Dar MM, Huang PS: Targeted anti-mitotic therapies: can we improve on tubulin agents? Nat Rev Cancer 2007;7:107-117.

-8 Lapenna S, Giordano A: Cell cycle kinases as therapeutic targets for cancer. Nat Rev Drug Discov 2009;8:547-566.

-9 Mu R, Wang YB, Wu M, Yang Y, Song W, Li T, Zhang WN, Tan B, Li AL, Wang N, Xia Q Gong WL, Wang CG, Zhou T, Guo N, Sang ZH, Li HY: Depletion of pre-mrna splicing factor cdc5l inhibits mitotic progression and triggers mitotic catastrophe. Cell Death Dis 2014;5:e1151.

-10 Bernstein HS, Coughlin SR: A mammalian homolog of fission yeast cdc5 regulates g2 progression and mitotic entry. J Biol Chem 1998;273:4666-4671.

- 11 Graub R, Lancero H, Pedersen A, Chu M, Padmanabhan K, Xu XQ, Spitz P, Chalkley R, Burlingame AL, Stokoe D, Bernstein HS: Cell cycle-dependent phosphorylation of human cdc5 regulates rna processing. Cell Cycle 2008; 7:1795-1803.

12 Nasmyth K, Nurse P: Cell division cycle mutants altered in dna replication and mitosis in the fission yeast schizosaccharomyces pombe. Mol Gen Genet 1981;182:119-124.

13 Ohi R, McCollum D, Hirani B, Den Haese GJ, Zhang X, Burke JD, Turner K, Gould KL: The schizosaccharomyces pombe cdc5+ gene encodes an essential protein with homology to c-myb. EMBO J 1994;13:471-483.

14 Ajuh P, Kuster B, Panov K, Zomerdijk JC, Mann M, Lamond AI: Functional analysis of the human cdc5l complex and identification of its components by mass spectrometry. EMBO J 2000;19:6569-6581.

15 Ajuh P, Sleeman J, Chusainow J, Lamond AI: A direct interaction between the carboxyl-terminal region of cdc5l and the wd40 domain of plrg1 is essential for pre-mrna splicing. J Biol Chem 2001;276:42370-42381.

-16 Boudrez A, Beullens M, Groenen P, Van Eynde A, Vulsteke V, Jagiello I, Murray M, Krainer AR, Stalmans W, Bollen M: Nipp1-mediated interaction of protein phosphatase-1 with cdc5l, a regulator of pre-mrna splicing and mitotic entry. J Biol Chem 2000;275:25411-25417.

17 Burns CG, Ohi R, Krainer AR, Gould KL: Evidence that myb-related cdc5 proteins are required for pre-mrna splicing. Proc Natl Acad Sci USA 1999;96:13789-13794.

-18 Burns CG, Ohi R, Mehta S, O'Toole ET, Winey M, Clark TA, Sugnet CW, Ares MJ, Gould KL: Removal of a single alpha-tubulin gene intron suppresses cell cycle arrest phenotypes of splicing factor mutations in saccharomyces cerevisiae. Mol Cell Biol 2002;22:801-815.

-19 McDonald WH, Ohi R, Smelkova N, Frendewey D, Gould KL: Myb-related fission yeast cdc5p is a component of a 40s snrnp-containing complex and is essential for pre-mrna splicing. Mol Cell Biol 1999;19:53525362.

20 Ohi MD, Link AJ, Ren L, Jennings JL, McDonald WH, Gould KL: Proteomics analysis reveals stable multiprotein complexes in both fission and budding yeasts containing myb-related cdc5p/cef1p, novel premrna splicing factors, and snrnas. Mol Cell Biol 2002;22:2011-2024.

-21 Tsai WY, Chow YT, Chen HR, Huang KT, Hong RI, Jan SP, Kuo NY, Tsao TY, Chen CH, Cheng SC: Cef1p is a component of the prp19p-associated complex and essential for pre-mrna splicing. J Biol Chem 1999;274:9455-9462. 


\section{Cellular Physiology Cell Physiol Biochem 2017;41:2475-2488 \begin{tabular}{l|l} 
DOI: 10.1159/000475916 & $\begin{array}{l}\text { O } 2017 \text { The Author(s). Published by S. Karger AG, Basel } \\
\text { www.karger.com/cpb }\end{array}$ \\
\hline
\end{tabular}}

Li et al.: CDC5L Promotes hTERT Expression

22 Zhang N, Kaur R, Akhter S, Legerski RJ: Cdc5l interacts with atr and is required for the s-phase cell-cycle checkpoint. EMBO Rep 2009;10:1029-1035.

23 Man TK, Lu XY, Jaeweon K, Perlaky L, Harris CP, Shah S, Ladanyi M, Gorlick R, Lau CC, Rao PH: Genome-wide array comparative genomic hybridization analysis reveals distinct amplifications in osteosarcoma. BMC Cancer 2004; 4:45.

24 Stewart SA, Hahn WC, O'Connor BF, Banner EN, Lundberg AS, Modha P, Mizuno H, Brooks MW, Fleming M, Zimonjic DB, Popescu NC, Weinberg RA: Telomerase contributes to tumorigenesis by a telomere lengthindependent mechanism. Proc Natl Acad Sci USA 2002;99:12606-12611.

25 Liu Z, Li Q Li K, Chen L, Li W, Hou M, Liu T, Yang J, Lindvall C, Bjorkholm M, Jia J, Xu D: Telomerase reverse transcriptase promotes epithelial-mesenchymal transition and stem cell-like traits in cancer cells. Oncogene 2013;32:4203-4213.

26 Ruden M, Puri N: Novel anticancer therapeutics targeting telomerase. Cancer Treat Rev 2013;39:444-456

27 Liu JL, Ge LY, Zhang GN: Telomerase activity and human telomerase reverse transcriptase expression in colorectal carcinoma. World J Gastroenterol 2006;12:465-467.

28 Gladych M, Wojtyla A, Rubis B: Human telomerase expression regulation. Biochem Cell Biol 2011;89:359376.

29 Kyo S, Takakura M, Fujiwara T, Inoue M: Understanding and exploiting htert promoter regulation for diagnosis and treatment of human cancers. Cancer Sci 2008;99:1528-1538.

-30 Deng WG, Jayachandran G, Wu G, Xu K, Roth JA, Ji L: Tumor-specific activation of human telomerase reverses transcriptase promoter activity by activating enhancer-binding protein-2beta in human lung cancer cells. J Biol Chem 2007;282:26460-26470.

-31 Bray F, Ren JS, Masuyer E, Ferlay J: Global estimates of cancer prevalence for 27 sites in the adult population in 2008. Int J Cancer 2013;132:1133-1145.

-32 Ramlee MK, Wang J, Toh WX, Li S: Transcription regulation of the human telomerase reverse transcriptase (htert) gene. Genes (Basel) DOI:10.3390/genes7080050.

-33 Upton HE, Chan H, Feigon J, Collins K: Shared subunits of tetrahymena telomerase holoenzyme and replication protein a have different functions in different cellular complexes. J Biol Chem 2017;292:217228.

Cifuentes-Rojas C, Shippen DE: Telomerase regulation. Mutat Res 2012;730:20-27. Merghoub N, El BH, Benbacer L, Gmouh S, Trentesaux C, Brassart B, Attaleb M, Madoulet C, Wenner T, Amzazi S, Morjani H, El MM: Tomentosin induces telomere shortening and caspase-dependant apoptosis in cervical cancer cells. J Cell Biochem DOI:10.1002/jcb.25826.

-37 Carkic J, Nikolic N, Radojevic-Skodric S, Kuzmanovic-Pficer J, Brajovic G, Antunovic M, Milasin J, Popovic B: The role of tert-clptm1l snps, htert expression and telomere length in the pathogenesis of oral squamous cell carcinoma. J Oral Sci 2016;58:449-458.

- 38 Jeon HS, Choi YY, Choi JE, Lee WK, Lee E, Yoo SS, Lee SY, Lee J, Cha SI, Kim CH, Park JY: Telomere length of tumor tissues and survival in patients with early stage non-small cell lung cancer. Mol Carcinog 2014;53:272-279.

-39 Pellatt AJ, Wolff RK, Herrick J, Lundgreen A, Slattery ML: Tert's role in colorectal carcinogenesis. Mol Carcinog 2013;52:507-513.

-40 Asghar U, Witkiewicz AK, Turner NC, Knudsen ES: The history and future of targeting cyclin-dependent kinases in cancer therapy. Nat Rev Drug Discov 2015;14:130-146.

-41 Schwartz GK, Shah MA: Targeting the cell cycle: a new approach to cancer therapy. J Clin Oncol 2005;23:9408-9421.

-42 Aida J, Izumo T, Shimomura N, Nakamura K, Ishikawa N, Matsuura M, Poon SS, Fujiwara M, Sawabe M, Arai T, Takubo K: Telomere lengths in the oral epithelia with and without carcinoma. Eur J Cancer 2010;46:430438.

-43 Martin JW, Chilton-MacNeill S, Koti M, van Wijnen AJ, Squire JA, Zielenska M: Digital expression profiling identifies runx2, cdc5l, mdm2, recql4, and cdk4 as potential predictive biomarkers for neo-adjuvant chemotherapy response in paediatric osteosarcoma. Plos One 2014;9:e95843.

-44 Mohammadi M, Goudarzi PK, Rahmani O, Kaghazian P, Yahaghi E, Taheriazam A, Ahmadi K: Evaluation of gene expression level of cdc5l and macc1 in poor prognosis and progression of osteosarcoma. Tumour Biol 2016;37:8153-8157. 